\title{
Viral loads in dual infection with HIV-1 and cytomegalovirus
}

\author{
Yuri S Boriskin, Mike Sharland, Ruth Dalton, Gill duMont, James C Booth
}

\begin{abstract}
Objective-A one year study of the relation between cytomegalovirus (CMV) and human immunodeficiency virus (HIV) viral loads in a cohort of children with vertically acquired HIV-1 infection.

Design-Comparative analysis of viral load measurements for CMV and HIV-1 in peripheral blood leucocytes (PBLs) of individual children in relation to age and clinical staging.

Methods-Nested polymerase chain reaction (PCR) was used to measure HIV-1 proviral DNA and CMV genomic DNA in PBLs of 56 children.

Results-The CMV load was highest in 0-2 year old HIV positive children with stage C disease (range, 1-7143 copies/100 ng DNA; median, 125) and was significantly lower in older children. Although higher in young children, HIV-1 viral load did not show the same marked reduction with age that is seen with CMV. Over a one year period, testing of serial samples for both viruses in a subgroup of children revealed a discordant relation between viral loads for CMV and HIV-1.

Conclusions-CMV viral load falls much faster than HIV viral load in dually infected children. Screening for clinical CMV disease is most likely to be of benefit in children under 2 years of age with stage $C$ disease. In the few children studied, levels of CMV and HIV replication appear to be independent.
\end{abstract}

(Arch Dis Child 1999;80:132-136)

Keywords: cytomegalovirus; human immunodeficiency virus; viral load

Between $40 \%$ and $80 \%$ of healthy prepubertal children are seropositive to human cytomegalovirus (CMV). High rates of coinfection have been reported in children vertically infected with human immunodeficiency virus (HIV) and $C M V,{ }^{1-5}$ with $30-60 \%$ excreting CMV and $18-39 \%$ of these children developing some clinical disease attributable to CMV. ${ }^{2}$

In infected adults, HIV viral load is predictive of progression to AIDS, ${ }^{67}$ and CMV DNAaemia predicts progression to disease, particularly retinitis. ${ }^{8}$ In younger children infected with HIV, the spectrum of CMV disease is less well established, partly because of the difficulties in performing accurate regular clinical screening for CMV retinitis. Our hypothesis was that measurement of CMV viral load might be useful in identifying those children most at risk of developing CMV disease. Because no previous data were available, we performed this study to determine the range of CMV viral loads found in children with HIV-1 infection.

CMV disease in children can follow a primary infection or reactivation of latent infection. There is some clinical evidence that children coinfected vertically with CMV and HIV may have a worse prognosis of their HIV disease. ${ }^{14}$ It is still not clear whether CMV acts as a cofactor in accelerating HIV pathogenesis or activates opportunistically, without clinical consequence, as a result of the general impairment in cellular immune responsiveness caused by HIV. ${ }^{124910}$ In a subgroup of children sequential blood samples were taken over one year, allowing the changes in CMV and HIV viral loads to be studied in relation to the children's ages and stages of HIV disease.

\section{Materials and methods}

STUDY POPULATION

Fifty six vertically acquired HIV-1 seropositive children all originating from sub-Saharan Africa and aged 0.5-13 years (median, 5.6 years; 10 under 2 years) who were attending the HIV family clinics of St George's, St Mary's, and St Thomas's Hospitals in London, were enrolled from July 1996. An extra 1-2 ml of blood was taken during routine outpatient phlebotomy when the child had no clinical signs of active intercurrent infection. All children were classified using the CDC clinical and immunological staging ${ }^{11}$ (fig 1), and underwent regular clinical and ophthalmological screening for CMV disease; $63 \%$ were receiving antiretroviral treatment, which consisted of various combinations of nucleoside analogues (AZT, ddI, ddC, 3TC, d4T). No child was taking protease inhibitors. All virological

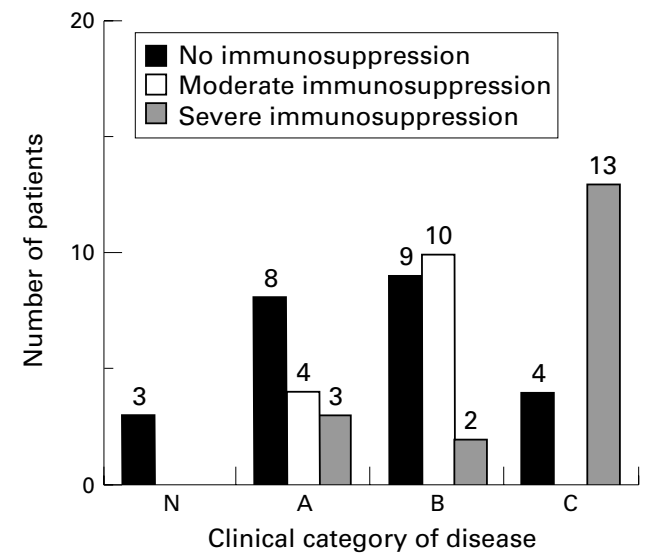

Figure 1 Distribution and number of patients according to CDC immunological and clinical staging of HIV disease. ${ }^{.1}$ 
investigations were blinded to the clinical details of the children. Monitoring for CMV IgG antibody was by in-house enzyme immunoassay. ${ }^{12}$

\section{NUCLEIC ACID ISOLATION}

Blood samples (1-2 $\mathrm{ml}$ in ethylene diaminetetra acetic acid) for testing by the polymerase chain reaction (PCR) were processed within 24 hours for cell associated CMV or HIV-1 proviral DNA. Peripheral blood mononuclear cells were extracted for total DNA by lysis with detergents followed by treatment with proteinase $\mathrm{K}^{13}$ The concentration of the extracted DNA, measured by fluorimetry, was adjusted to $100 \mathrm{ng} / \mu \mathrm{l}$ in the extraction buffer.

\section{SEMIQUANTITATIVE DUPLEX PCR FOR HIV-1 AND} CMV DNA

Nested PCR reactions for amplifying a 168 base pair (bp) DNA fragment from the mtrII immediate-early region of the CMV genome $\mathrm{e}^{14}$ and a $360 \mathrm{bp}$ fragment of the HIV-1 env V3 region $^{13}$ were performed in the same $50 \mu 1$ reaction volume. The details of the primer sequences, the composition of the PCR reaction mixture, and the cycling conditions were as described previously. ${ }^{13}{ }^{14}$ The HIV-1 and CMV DNA amplicons were resolved in a $6 \%$ mini polyacrylamide gel from which the appropriate specific bands were eluted for direct sequencing ${ }^{15}$ and for CMV DNA restriction analysis. ${ }^{16}$ Samples found to be negative by the HIV PCR were retested by PCR against primers specific for the "housekeeping" histidyl tRNA synthetase (HRS) gene ${ }^{14}$; these primers were not included routinely because the product size ( $360 \mathrm{bp}$ ) was the same as with the HIV env primers.

The sensitivity of the CMV mtrII PCR was of the order of one copy of specific plasmid DNA, ${ }^{16}$ as was that of the HIV-1 env PCR ${ }^{13}$ when determined using the MRC ADP PCR reference reagents set (No. ADP956). ${ }^{17}$

The duplex CMV-HIV PCR was performed on serial 10-fold dilutions of DNA, in duplicate, and the number of virus copies for either virus was calculated for each $100 \mathrm{ng}$ of total cell DNA, on the assumption that the signal at the end point of the titration emanated from one copy of viral DNA being added at the start of the reaction. The number of $\mathrm{CD} 4$ positive cells was not measured on every occasion and, therefore, the number of viral copies was expressed for each $100 \mathrm{ng}$ of leucocyte DNA, which is an equivalent of 15000 cells. Preliminary testing established that duplex or monoplex PCR, when performed using the same primers, gave similar end point values for either virus on titrating blood samples that contained high levels of DNA for both viruses; this showed that there was no significant interference between the two PCR reactions when these were proceeding simultaneously. At the time of our study, the improved Amplicor HIV-1 monitor RNA PCR test using the primers SK 145/SK151 was not available, and the most comprehensive set of data for HIV viral loads throughout the study was that obtained by the nested PCR for proviral DNA. Com- parison of the concentrations of HIV-1 env DNA and HIV-1 gag RNA in 14 children (data not shown) showed a close correlation as noted by others. ${ }^{18} 19$ Therefore, all data presented were obtained by using duplex nested PCR for CMV DNA and HIV-1 proviral DNA.

Statistical analysis was performed by the Kruskal-Wallis test using the MultiStat application for the Macintosh; this compares median values for groups of non-normally distributed data.

\section{Results}

VIRAL LOAD MONITORING FOR HIV AND CMV INFECTION

The specificity of the PCR assay was tested in two groups of healthy children who served as controls for our study. The first group contained $18 \mathrm{HIV}$ antibody negative children, aged $0.1-2$ years $(n=12)$ and $4-9$ years $(n=6)$, of HIV positive African women. All 18 children were negative by PCR for HIV-1 DNA; nine were positive for antibody to CMV and two of these were also positive for CMV DNA.

The other control group contained 44 healthy children, aged $0.1-14$ years (median, 5.6) whose blood samples were obtained during routine surgical procedures; 10 were positive for antibody to CMV and one of these was positive also by CMV PCR. The levels of CMV DNA recorded in the three HIV negative, CMV PCR positive children were in all cases one copy/100 ng of total DNA.

Our study group contained 56 HIV-1 antibody positive children; 54 of these were positive for HIV proviral DNA. Of the two subjects who gave discordant results, one was a 7 year old boy who had been tested by HIV PCR for DNA three times every year since 1992, including twice during the course of our study and who, despite having CD4 positive cell counts of $\sim 1000$, had remained consistently negative for HIV DNA, yet weakly positive for HIV RNA. The other was an 8 year old girl (CD4 positive cell counts, 500-600 during our study) who had tested positive by HIV PCR for DNA in 1993, but had been found to be PCR negative in 1996-97, yet positive for HIV RNA. In these two patients, the discordant results between the PCR for HIV-1 DNA and HIV-1 RNA could be the result of the higher sensitivity of the HIV-1 RNA PCR, probably because the gag region is relatively more conserved than the highly variable env sequence of HIV-1, especially in viral isolates of African origin.

Forty five of the $56 \mathrm{HIV}-1$ antibody positive children were positive for CMV IgG. Among the 11 who were repeatedly CMV antibody negative, six gave negative results for CMV DNA by PCR, and the other five were weakly positive (1-2 copies of CMV DNA/100 ng of total DNA in three patients; six and 10 copies in the other two patients).

PATTERNS OF HIV DNA AND CMV DNA VIRAL LOAD CMV and HIV viral loads were compared in relation to the age of the child at testing. All 56 children were split in to two groups: one group 


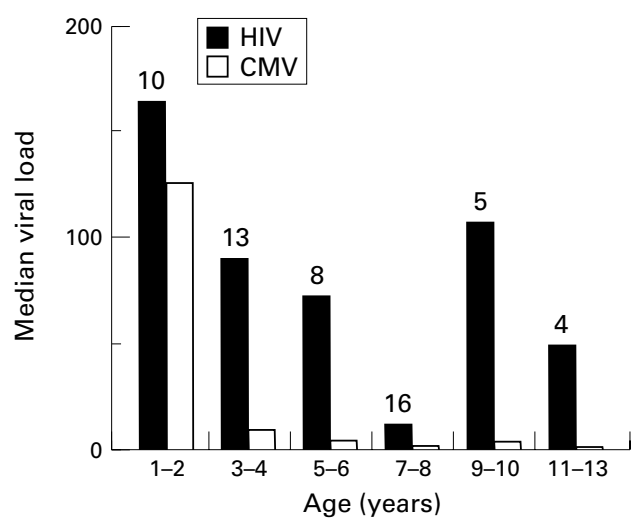

Figure 2 Median HIV-1 and CMV viral loads (copies of viral DNA/100 ng of total DNA) in children of different age groups. The numbers above the bars are numbers of children in each age group.

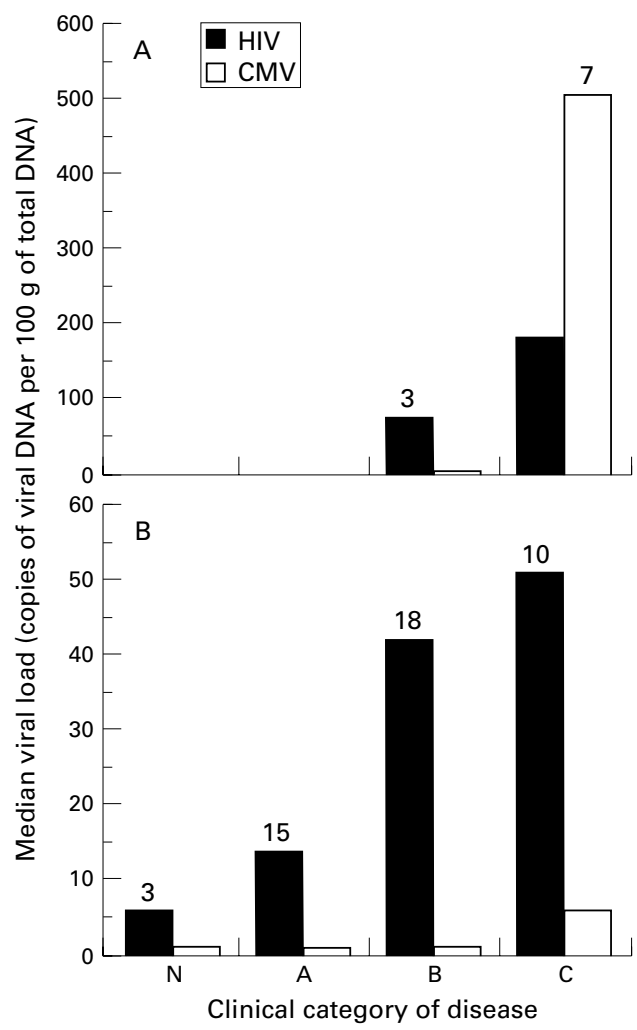

Figure 3 Relation between $H I V-1$ and $C M V$ viral load related to the CDC clinical category of HIV disease in children $(A)$ under 2 and $(B)$ over 2 years of age.

comprising children aged over 2 years $(n=46)$ and the other those under 2 years $(n=10)$; the latter group contained three children in clinical stage $B$ and seven in stage $C$. The viral loads for CMV DNA in the $0-2$ years age group (0-7143 copies/100 ng DNA; median, 125) was significantly higher $(\mathrm{p}=0.0028)$ than in the older age group (fig 2), whereas the median viral loads for HIV proviral DNA were not significantly different between the two groups. The pattern of CMV and HIV viral loads by age of the child is shown in fig 2 .

Because the levels of CMV were higher in the younger children, the two groups of children aged over and under 2 years were compared to see if there was any association between the viral loads for HIV and CMV and the child's clinical staging. For those aged under 2 years, high viral loads for CMV were significantly associated with clinical stage C $(p=0.014)$. However, although the HIV viral loads in this group were clearly higher in children in clinical stage $\mathrm{C}$, the association between HIV viral load and clinical staging was not significant (fig 3).

To examine more closely the relation between levels of viraemia for CMV and HIV in younger children, longitudinal testing of a subgroup of children under 2 years of age for both viruses was carried out over the one year period. The results for eight such patients are presented in fig 4 . In four of these patients (A, C, G, and $\mathrm{H}$ ) high levels of HIV DNA were detected initially but declined and were replaced by high levels of CMV DNA; in the other four patients (B, D, E, and F) the converse was true, with CMV DNA levels predominating early on but eventually being superseded by HIV. Only one 1 year old boy had clinical CMV disease during our study. Severe (zone 3) bilateral CMV retinitis was detected during routine screening; his peak CMV viral load was 500 CMV DNA copies/ $100 \mathrm{ng}$ DNA and this showed no decline in response to ganciclovir treatment (fig $4 \mathrm{H}$ ).

\section{Discussion}

Several studies have confirmed the relation between HIV-1 viral load and disease progression in both adults ${ }^{67}$ and children, ${ }^{20-23}$ and have indicated that CMV DNAaemia levels are predictive for the development of CMV end organ disease in cases of adult HIV infection. ${ }^{3824}$ Quantitative CMV PCR has shown a strong association between higher baseline copy number and the earlier development of CMV disease in a recent anti-CMV drug study. ${ }^{25}$

In our study, most of the HIV infected children appeared to be infected with CMV, as indicated by the detection of CMV IgG antibody and viral DNA. Five children were CMV antibody negative or equivocal, while being weakly positive for CMV DNA. A similar discrepancy between CMV serology and PCR has been noted previously in a group of heart transplant patients. ${ }^{16}$ Interestingly, this suggests that there might be a qualitative or quantitative effect of immunosuppression on CMV antibody production in some patients. For example, a response comprising mainly low avidity antibody could give a false negative result by standard antibody assay.

The cross sectional component of this study detected no clear association between a high CMV viral load and the development of CMV end organ disease because of the low frequency of CMV retinitis in the study group (one of eight patients with high CMV load; none of four patients who died). The very high levels of CMV viral load in the first 2 years of life, and the rapid apparent subsequent decline suggest that sequential long term testing for CMV viral load will not be useful in predicting clinical CMV disease in HIV infected children. The introduction of "highly active antiretroviral therapy" (HAART) in adults has recently been 


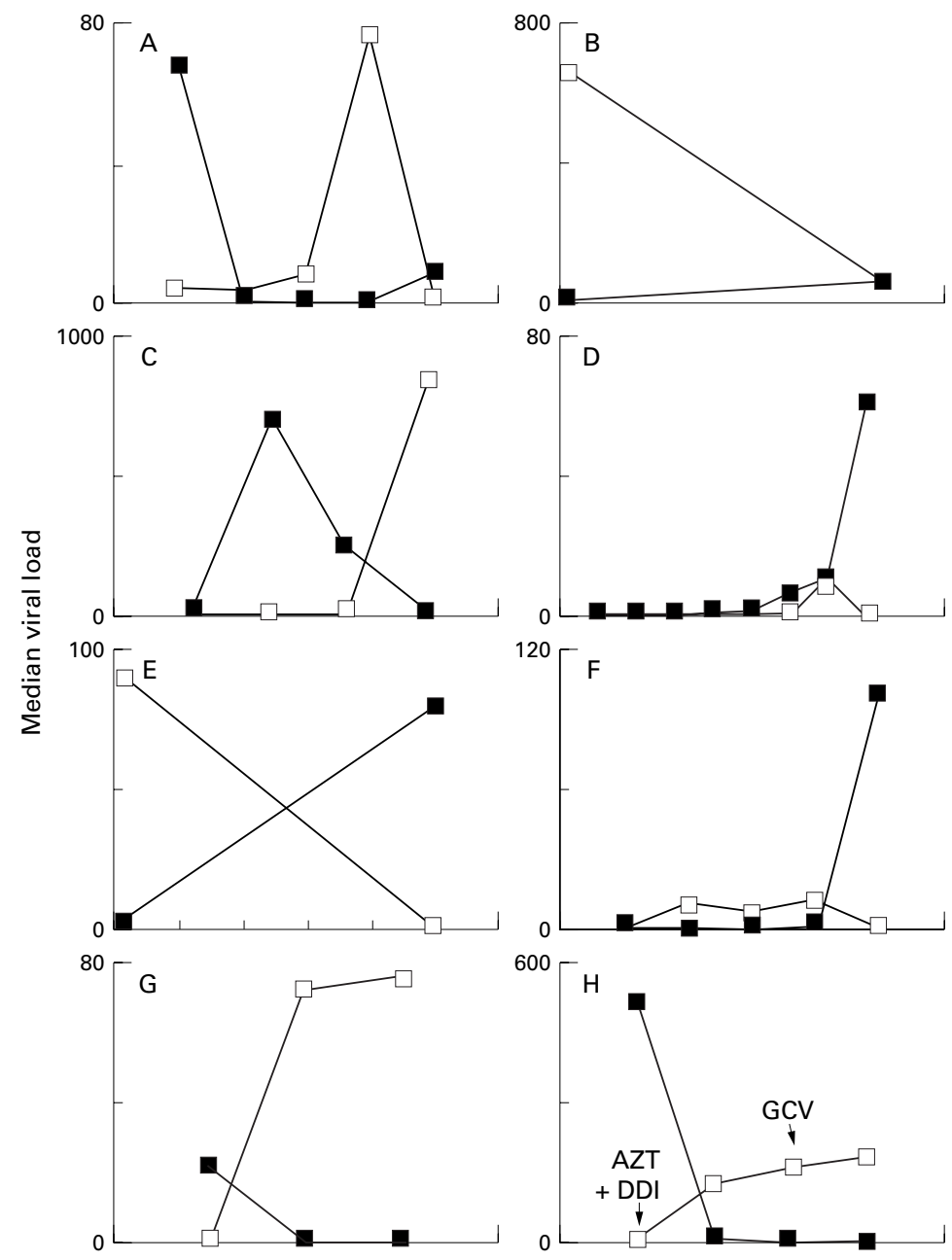

Intervals of sampling (months)

Figure 4 Discordant HIV-1/CMV load patterns (copies of viral DNA/100 ng total $D N A$ ) in eight individual patients under 2 years of age, determined by duplex PCR (similar results were obtained by monoplex PCR for each virus alone). Closed symbols, HIV-1 load; open symbols, CMV load. Patients mean CD4 positive lymphocyte count over study period $\left(\times 10^{9}\right)$ : (A) 0.5; (B) 1.48; (C) 0.6; (D) 1.77; (E) 1.43; (F) 0.8; (G) 0.95; (H) 0.3 .

associated with a dramatic reduction in the frequency of CMV disease.

In agreement with earlier studies, ${ }^{22} 23$ the highest HIV-1 viral loads were found in younger children in clinical stage C. High CMV viral loads were found only in the $0-2$ year old age group, remaining low in the older age groups. All the infants in our study had presented with rapidly progressive disease and high HIV viral loads. In the absence of any infants followed prospectively from birth it is not possible to identify whether the age or stage of HIV disease is the principal explanatory variable for determining the high CMV load.

An unexpected finding was that the levels of HIV-1 and CMV DNA showed no consistent association in individual patients. In the blood, latent CMV and HIV-1 occupy different cell compartments. For CMV these are usually monocytes and polymorphs, ${ }^{26-28}$ and other white blood cell types in cases of active infection. ${ }^{29}$ For HIV-1, proviral DNA is restricted predominantly to CD4 positive lymphocytes. ${ }^{30}$ For these reasons, HIV and
CMV coinfection of white blood cells is considered to be an unlikely event, ${ }^{31}$ and this is borne out in our small study by the finding that high viral loads for these two viruses appeared to be mutually exclusive. However, HIV and CMV might interact in tissues such as lymphoid organs, either by coinfecting the same cell type (for example, macrophages), or by a CMV encoded superantigen that enhances $\mathrm{HIV}-1$ replication in V $\beta 12$ positive, CD4 positive cells. ${ }^{32}$ Why levels of cell associated HIV-1 and CMV in the peripheral blood appeared to be mutually exclusive is not clear, but might reflect the differential effects of cytokines on virus replication. ${ }^{33}$

Several conclusions can be drawn from our results. First, because high level CMV DNAaemia is most common in the $0-2$ year old age group of HIV-1 vertically infected children, it implies that the CMV infection in this group of patients is the result of intrauterine or perinatal primary infection, rather than reactivation of latent CMV. Because most CMV infected children with a high CMV load were in clinical stage C of their HIV disease, this suggests that early, possibly intrauterine, onset of infection with HIV, when followed by rapid development of immunosuppression, predisposes these patients to systemic CMV infection. In the absence of widespread availability of CMV viral load measurements, it is this group of patients who would probably benefit most from regular ophthalmological surveillance.

In children over 2 years there was no clear association between CMV load and stage of the HIV disease. High levels of CMV in young children with immature immune systems might be associated with impaired cellular immunity to $\mathrm{CMV}^{34}$ In older children, whose immune system is able to bring CMV under control, ${ }^{34}$ the most important factor in progression to CMV disease is probably the rate at which HIV infection is able to overwhelm the overall immune response. The temporal relation between the levels of HIV-1 infection and CMV and the discordant HIV/CMV load pattern in individual patients suggests that both viruses replicate independently of one another, and that CMV is not acting as a major promoter of HIV disease progression.

We thank Drs H Lyall, G Tudor-Williams, and J Dodge from St Mary's Hospital, and S O'Shea of St Thomas's Hospital for helping with patients' specimens. The HIV-1-specific primers and the PCR reference reagents were supplied by the Medical Research Council AIDS directed programme. This work was sponsored by the South Thames Regional Health Authority HIV/AIDS Ringfenced Allocation 1996-1997

1 Frenkel LD, Gaur S, Tsolla M, Scudder R, Howell R, Kesarwala $\mathrm{H}$. Cytomegalovirus infection in children with AIDS. Rev Infect Dis 1990;12:5820-6.

2 Chandwani S, Kaul A, Bebenroth D, et al. Cytomegalovirus infection in human immunodeficiency virus type 1 -infected children. Pediatr Infect Dis f 1996;15:310-14

3 Nigro G, Krzysztofiak A, Gattinara GS, et al. Rapid progression of HIV disease in children with cytomegalovirus DNAemia. AIDS 1996;10:1127-33.

4 Kitchen BJ, Engler HD, Gill VJ, et al. Cytomegalovirus infection in children with human immunodeficiency virus infection. Pediatr Infect Dis f 1997;16:358-63.

5 Scott GB, Buck BE, Leterman JG, Bloom FL, Parks WP. Acquired immunodeficiency syndrome in infants. N Engl $\dot{f}$ Med 1984;310:76-81.

6 Mellors JW, Rinaldo CR, Gupta P, White RM, Todd JA, Kingsley LA. Prognosis in HIV-1 infection predicted by the quantity of virus in plasma. Science 1996;272:1167-70. 
7 O'Brien WA, Hartigan PM, Martin D, et al. Changes in plasma HIV-1 RNA and CD4 lymphocyte counts and the
risk of progression to AIDS. $N$ Engl $f$ Med $1996 ; 334: 426-$ 31 .

8 Spector SA, Merrill R, Wolf D, Daukner WM. Detection of human cytomegalovirus in plasma of AIDS patients during acute visceral disease by DNA amplification. $f$ Clin Microbiol 1992;30:2359-65.

9 Griffiths PD. Herpesviruses and AIDS. F Antimicrob Chem other 1996;37:87-95.

10 Domachowske JB. Pediatric human immunodeficiency virus infection. Clin Microbiol Rev 1996;9:448-68.

11 Centers for Disease Control and Prevention. 1994 revised classification system for human immunodeficiency virus infection in children less than 13 years of age. Morbid Mor tal Weekly Rep 1994; 43:1-19.

12 Booth JC, Hannington G, Bakir T, et al. Comparison of enzyme-linked immunosorbent assay, radioimmunoassay, complement fixation, anti-complement fluorescence and passive haemagglutination techniques for detecting cypassive haemagglutination techniques for detecting cy-
tomegalovirus IgG antibody. $\mathcal{F}$ Clin Pathol 1982;35:1345-8.

13 Boriskin YS, Booth JC, Fernando S, et al. The detection of HIV-1 proviral DNA by PCR in clotted blood specimens. I Virol Methods 1995;52:87-94.

14 Fernando S, Booth J, Boriskin Y, et al. Association of cytomegalovirus infection with post-transplantation cardiac rejection as studied using the polymerase chain reaction. F Med Virol 1994;41:108-13.

15 Boriskin YS, Booth JC, Yamada A. Sequence variation within the carboxyl terminus of the nucleoprotein gene of mumps virus strains. Clin Diagn Virol 1994;2:79-85.

16 Boriskin YS, Booth JC, Corbishley CM, et al. Human cytomegalovirus and acute rejection after heart transplantation are not directly associated. $\mathcal{F}$ Med Virol 1996;50:5970 .

17 Bootman JS, Kitchin PA. An international collaborative study to assess a set of reference reagents for HIV-1 PCR. $\mathcal{F}$ Sirol Methods 1992;37:23-42.

18 Verhofstede C, Reniers S, Van Wanzele F, Plum J. Evaluation of proviral copy number and plasma RNA level
as early indicators of progression in HIV-1 infection: correas early indicators of progression in HIV-1 infection: correlation with virological and
disease. AIDS 1994;8:1421-7.

19 Sei S, Akiyoshi H, Bernard J, et al. Dynamics of virus versus host interaction in children with human immunodeficiency virus type 1 infection. F Infect Dis 1996;173:485-90.

20 Palumbo PE, Kwok S, Waters S, et al. Viral measurement by polymerase chain reaction-based assays in human immunodeficiency virus-infected infants. F Pediatr 1995;126: 592-5.

21 McIntosh K, Shevitz A, Zaknun D, et al. Age- and time-related changes in extracellular viral load in children infected with human immunodeficiency virus. Pediatr Infect Dis f 1996;15:1087-91.
22 Mofenson LM, Korelitz J, Meyer III WA, et al. The relationship between serum human immunodeficiency virus type 1 ship between serum human immunodeficiency virus type 1
(HIV-1) RNA level, CD4 lymphocyte percent, and (HIV-1) RNA level, CD4 lymphocyte percent, and long-term mortality risk in
Dis 1997;175:1029-38.

23 Tetali S, Abrams E, Bakshi S, Paul M, Oyaizi N, Pahwa S. Virus load as a marker of disease progression in HIV-infected children. AIDS Res Hum Retroviruses 1996; 12:669-75.

24 Dodt KK, Jacobsen PH, Hoffman B, et al. Development of cytomegalovirus (CMV) disease may be predicted in HIVinfected patients by CMV polymerase chain reaction and the antigenemia test. AIDS 1997;11:F21-8.

25 Feinberg J. The status of CMV viral load testing. AIDS Clinical Care 1996;8:81.

26 Turtinen LW, Saltzman R, Jordan MC, Haase AT. Interactions of human cytomegalovirus with leukocytes in vivo: analysis by in situ hybridization. Microb Pathog 1987; 3:287-97.

27 Soderberg C, Larsson S, Bergstedt-Lindqvist S, Moller E. Definition of a subset of human peripheral blood mononuclear cells that are permissive to human cytomegalovirus infection. F Virol 1993;67:3166-75.

28 Mitchell BM, Leung A, Stevens JG. Murine cytomegalovirus DNA in peripheral blood of latently infected mice is detectable only in monocytes and polymorphonuclear leukocytes. Virology 1996;223:198-207.

29 Von Laer D, Serr A, Meyer-Konig U, Kirste G, Hufert FT, Haller O. Human cytomegalovirus immediate early and late transcripts are expressed in all major leukocyte populalate transcripts are expressed in all major leuko
tions in vivo. $\mathcal{A}$ Infect Dis 1995;172:365-70.

30 Shen Y, Rudnik J, Cassol S, et al. Blood monocytes from most human immunodeficiency virus type 1 -infected patients do not carry proviral DNA. Clin Diagn Lab Immunol 1994;1:531-7.

31 Bertram S, Hufert FT, Van Lunzen J, Von Laer D. Coinfection of individual leukocytes with human cytomegalovirus and human immunodeficiency virus is a rare event in vivo. 7 Med Virol 1996;49:283-8.

32 Dobrescu D, Ursea B, Pope M, Asch AS, Posnett DN. Enhanced HIV-1 replication in VB12 cells due to human cytomegalovirus in monocytes: evidence for a putative herpesvirus superantigen. Cell 1995;82:753-63.

33 Hatch WC, Freedman AR, Boldt-Houle DM, Groopman JE, Terwilliger EF. Differential effects of interleukin-13 on cytomegalovirus and human immunodeficiency virus infection in human alveolar macrophages. Blood 1997;89: 3443-50.

34 Pass RF, Dworsky ME, Whitley RJ, August AM, Stagno S, Alford CA. Specific lymphocyte blastogenic responses in children with cytomegalovirus and herpes simplex virus infections acquired early in infancy. Infect Immun 1981;34: $166-70$. 